\title{
Microbiological Quality Assessment of Ready-to-eat Vegetables in Dubrovnik-Neretva County, Croatia
}

\author{
Ivana Ljevaković-Musladin ${ }^{1, *}$, Mato Lakić ${ }^{1}$, Lidija Kozačinski ${ }^{2}$ \\ ${ }^{1}$ Environmental Health Department, Public Health Institute of Dubrovnik-Neretva County, Croatia \\ ${ }^{2}$ Department of Hygiene, Technology and Food Safety, Faculty of Veterinary Medicine, Croatia
}

Copyright $\bigcirc 2019$ by authors, all rights reserved. Authors agree that this article remains permanently open access under the terms of the Creative Commons Attribution License 4.0 International License

\begin{abstract}
The aim of this study was to assess the microbiological quality of ready-to-eat vegetables offered by local food business operators in Dubrovnik-Neretva County in the period of 2011-2018. Vegetables have been randomly selected and sampled in hotels, restaurants and retail. Analysis has been assessed according to Regulation (EC) No 2073/2005 on microbiological criteria for foodstuffs and Croatian National Guidelines on microbiological criteria for foodstuffs [1,2]. A total of 243 samples were analysed for Enterobacteriaceae, E. coli, Coagulase-positive staphylococci, sulphite-reducing Clostridia, yeasts/molds, Listeria monocytogenes and the presence of Salmonella spp., using respective ISO methods. The results showed that $136(56 \%)$ samples were unsatisfactory, mostly because of the high levels of yeasts/molds and Enterobacteriaceae. Unsatisfactory levels of yeasts/molds was found in $105(43.2 \%)$ samples, Enterobacteriaceae in $96 \quad(39.5 \%)$ samples, Coagulase-positive staphylococci in $12(4.9 \%)$ samples and $E$. coli in $4(1.6 \%)$ samples. Among these 136 samples concentration rang of Enterobacteriaceae, Escherichia coli, Coagulase-positive staphylococci and yeasts/molds were 3.20-6.48, 3.60-5.81, 3.26-4.65 and 3.08-5.48 $\log \mathrm{CFU} / \mathrm{g}$, respectively. Sulphite-reducing Clostridia, Listeria monocytogenes and Salmonella spp. were not detected in any of the samples. The most contaminated vegetables were arugula and lettuce, followed by cabbage and salad mix. All unsatisfactory samples were either sliced or grated. In 2017, 11 samples of packaged ready-to-eat leafy green vegetables were also analyzed. 7 out of 11 samples $(63.6 \%)$ were unsatisfactory due to contamination with Enterobacteriaceae (4-5 $\log \mathrm{CFU} / \mathrm{g}$ ), although the products were labelled as ready-to-eat with no need for washing. Although pathogens weren't isolated, our study showed poor microbiological quality of vegetables and brings certain concern regarding the safety of these products. Proper washing and adequate hygiene during preparation are a "must have" prerequisite for food safety. More studies are needed regarding microbiological quality of packaged ready-to-eat vegetables, since their

mislabelling is giving a customer incorrect information. Keywords Ready-to-eat Vegetables, Microbiological Quality, Food Safety, Public Health

\section{Introduction}

Fruits and vegetables are important component of a healthy and balanced diet. In 2005, the World Health Organization (WHO) in association with the Food and Agriculture Organization of the United Nations (FAO) started the international campaign for increasing the consumption of fruits and vegetables with the main purpose of health improvement and prevention of cardiovascular diseases and some types of cancer [3]. Since 1990, the global harvesting area for fruits and vegetables has progressively increased. According to FAOSTAT [3], the harvest area for lettuce and chicory increased by $218 \%$ and for spinach increased by $300 \%$ in the period 1986 to 2006. From 1980 to 2004, the global production per annum (p.a.) of fruit and vegetables grew by $94 \%$ [3].

Increased consumption, large-scale production and global distribution led to increase in number of foodborne diseases worldwide, attributed to fresh produce as vehicles [4-6]. Fresh vegetables contain their natural epiphytic microflora which is mostly non-pathogenic. However, during growth, harvesting, processing, packaging, handling and retail there is a risk of microbial contamination with pathogenic microorganisms. The sources of contamination include irrigation water, wild birds, animals, organic fertilizers, washing water during processing, handling by workers and contact with contaminated surfaces. Irrigation water has been considered to be the major source of enteric pathogens [7].

Fresh produce has been implicated in foodborne diseases worldwide. Leafy greens, such as lettuce, spinach, and fresh herbs, e.g. parsley and basil, are more frequently
\end{abstract}


implicated in human illness [4]. Epidemiological data shows that major health risks from fresh vegetables include: bacteria (E. coli O157:H7, Salmonella spp., Listeria monocytogenes, Campylobacter spp., Shigella spp.), viruses (norovirus, rotavirus, hepatitis A virus) and parasites (Giardia, Echinococcus, Cryptosporidium, Entamoeba, Cyclosporidium) [4-6]. According to EFSA, the highest number of foodborne outbreaks was reported for the combination of norovirus and leafy greens eaten raw as salads, followed by the combination of Salmonella and leafy greens eaten raw as salad $[5,6]$. According to the same EFSA report, the top ranking food/pathogen combination, besides Salmonella spp./ leafy greens, was (in equal rank) Salmonella spp./bulb and stem vegetables, Salmonella spp./tomatoes, Salmonella spp./melons, and pathogenic Escherichia coli and fresh pods, legumes or grain [5].

The highest number of human cases was reported for pathogenic Escherichia coli in Germany in 2011. Large VTEC O104 outbreak was associated with sprouted fenugreek seeds (3,793 human cases, 2,353 hospitalizations and 53 deaths) [5].

The most recent multistate outbreak of E. coli $\mathrm{O} 157: \mathrm{H} 7$ infection, which lasted from April to June 2018 in the USA was linked to romaine lettuce. 210 people from 36 states were infected, 96 were hospitalized, including 27 people who developed hemolytic uremic syndrome specific for E. coli $\mathrm{O} 157: \mathrm{H} 7$, five deaths were reported. Canal water was the most likely source of contamination [8].

Several outbreaks of campylobacteriosis caused by consumption of leafy vegetables and salads [9], lettuce [10], sprouts and cabbage [11] have raised interest in the campylobacteriosis risk assessment of raw vegetables. Recently published systemic review and meta-analysis of the prevalence of Campylobacter in vegetables, fruits, and fresh produce estimated the prevalence to be $0.53 \%$. Despite the low prevalence, consumption of raw vegetables is risky because no treatment is used to inactivate the pathogen and the high level of consumption of these products raises total risk of infection [12].

Another recent outbreak (July-September 2018) also in the USA was Cyclospora cayetanensis infections linked to salad mix. 511 people were infected from 15 states and New York City, no deaths were reported. Laboratory analysis of unused romaine lettuce and carrot mix, which were the ingredients of the salad mix, confirmed the presence of Cyclospora [13].

Besides epidemiological data, numerous prevalence studies have shown evidence that indicator and pathogenic bacteria are present on fresh and ready-to-eat vegetables.

The aim of this study was to assess the microbiological quality of ready-to-eat (RTE) vegetables offered by local food business operators in Dubrovnik-Neretva County in the period of 2011-2018.

\section{Materials and Methods}

\subsection{Sampling}

Vegetables have been randomly selected and sampled in hotels, restaurants and retail in Dubrovnik-Neretva County during the period of 2011-2018. Sampling targeted vegetables already prepared for consumption (sliced, grated, mixed). Both packaged and unpackaged products were sampled. Sprouted seeds were not included in assessment since they are not very popular in our country, hence very rarely present in our local food offer.

\subsection{Analysis}

All samples were analyzed for Salmonella spp., Listeria monocytogenes, Enterobacteriaceae, E. coli, Coagulase-positive staphylococci, sulphite-reducing Clostridia, yeasts and molds, according to criteria from Regulation (EC) No 2073/2005 on microbiological criteria for foodstuffs and Croatian National Guidelines on microbiological criteria for foodstuffs. Samples were examined in ISO 17025 accredited laboratory of the Public Health Institute of Dubrovnik-Neretva County.

\section{Detection of Salmonella spp}

Analytical procedure was performed according to the International Standard method ISO 6579 for detection of Salmonella spp. $25 \mathrm{~g}$ of sample was analyzed after homogenization in a Stomacher for $2 \mathrm{~min}$. Media used in this procedure include: Buffered Peptone Water, Rappaport-Vassiliadis Soy (RVS) Broth medium, Muller-Kaufmann Tetrathionate (MKTTn) Broth medium, XLD agar and Brilliant Green agar (all Oxoid). In the case of presence of presumptive colonies on XLD agar and/or Brilliant green agar, five colonies were picked for biochemical and serological confirmation. Tests described in ISO 6579-1:2017 were used for biochemical identification. For serological confirmation Anti-serum Salmonella Omnivalent Omni-O, Anti-serums Salmonella Polyvalent OMA, Salmonella Polyvalent OMB and Salmonella Polyvalent OMC (Bio-Rad) were used. Positive isolates were sent to National Reference Laboratory for further serological identification.

\section{Detection of Listeria monocytogenes}

Analytical procedure was performed according to the International Standard method ISO 11290-1 for detection of Listeria monocytogenes. $25 \mathrm{~g}$ of sample was analyzed after homogenization in a Stomacher for $2 \mathrm{~min}$. Media used in this procedure include: Listeria Half Fraser Broth medium, Fraser Broth medium, Chromogenic Listeria Agar (OCLA) medium and Palcam Agar (all Oxoid). ). In the case of presence of presumptive colonies on OCLA medium and/or Palcam Agar, five colonies were picked for biochemical confirmation which was carried out according 
to ISO $11290-1$.

Enumeration of L. monocytogenes, Enterobacteriaceae, E. coli, Coagulase-positive staphylococci, Sulphite-reducing Clostridia and Yeasts/Molds

Analytical procedures were performed according to the International Standard methods: ISO 11290-2 for enumeration of Listeria monocytogenes, ISO 21528-2 for enumeration of Enterobacteriaceae, ISO 16649-2 for enumeration of Escherichia coli, ISO 6888-1 for enumeration of Coagulase-positive staphylococci, ISO 15213 for enumeration of sulphite-reducing Clostridia and ISO 21527-1 for enumeration of yeasts and molds. A $10 \mathrm{~g}$ of sample was homogenized in a Stomacher for $2 \mathrm{~min}$. 10 -fold serial dilutions were prepared and then subsequently processed for enumeration of specified microorganisms using OCLA agar, VRBG agar, TBX agar, Baird-Parker Agar, TSC agar and DRBC agar (all Oxoid), respectively. Incubations as well as identifications were performed according to specific ISO standard method.

\section{Results and Discussion}

Regulation (EC) No 2073/2005 on microbiological criteria for foodstuffs defines mandatory microbiological criteria. Listeria monocytogenes and Salmonella spp. are listed as safety criteria. On the other hand, Escherichia coli is listed as process hygiene criteria. All other microorganisms were analyzed according to Croatian
National Guidelines on microbiological criteria for foodstuffs. These guidelines (Table 1) serve as a valuable tool for microbiological quality assessment and risk management.

A total of 243 samples were analyzed. The results in Table 2 shows that $136(56 \%)$ samples did not meet the microbiological criteria (labelled as unsatisfactory samples), due to high levels of yeasts/molds and Enterobacteriaceae. As shown in Table 3, unsatisfactory levels of yeasts/molds were found in $105(43.2 \%)$ samples and Enterobacteriaceae in 96 (39.5\%) samples, Coagulase-positive staphylococci in $12(4.9 \%)$ samples and E. coli in $4(1.6 \%)$ samples. Among unsatisfactory samples concentration range of Enterobacteriaceae, Escherichia coli, Coagulase-positive staphylococci and yeasts/molds were $3.60-5.81,3.20-6.48,3.26-4.65$ and 3.08-5.48 log CFU/g, respectively. Sulphite-reducing Clostridia, Listeria monocytogenes and Salmonella spp. were not detected in any of the samples.

The most contaminated vegetables were arugula and lettuce, followed by cabbage, salad mix and cucumbers. We presume that such results for arugula and lettuce were due to insufficient washing process which was probably minimized since leafy greens are very sensitive to prolonged water exposure. All unsatisfactory samples were either sliced or grated. Both actions can introduce additional amount of microorganisms spread by utensils or dirty hands. The best microbiological quality was found for tomatoes.

Table 1. Microbiological criteria for RTE vegetables

\begin{tabular}{|c|c|c|c|}
\hline Legal act & Type of criteria & Microorganism & Limits \\
\hline \multirow{2}{*}{$\begin{array}{c}\text { Regulation (EC) No } \\
2073 / 2005\end{array}$} & $\begin{array}{c}\text { Mandatory } \\
\text { Safety criteria }\end{array}$ & Salmonella spp. & Absence in $25 \mathrm{~g}$ \\
\cline { 2 - 4 } & $\begin{array}{c}\text { Mandatory } \\
\text { Process hygiene criteria }\end{array}$ & Listeria monocytogenes & $100 \mathrm{cfu} / \mathrm{g}$ \\
\hline \multirow{2}{*}{$\begin{array}{c}\text { Croatian National } \\
\text { Guidelines on } \\
\text { microbiological criteria } \\
\text { for foodstuffs }\end{array}$} & $\begin{array}{c}\text { Process hygiene criteria } \\
\text { guidelines }\end{array}$ & Enterobacteriaceae & $1000 \mathrm{cfu} / \mathrm{g}$ \\
\cline { 2 - 4 } & Coagulase-positive staphylococci & $100 \mathrm{cfu} / \mathrm{g}$ \\
\cline { 2 - 4 } & Sulphite-reducing Clostridia & $1000 \mathrm{cfu} / \mathrm{g}$ \\
\hline
\end{tabular}

Table 2. Results of the microbiological examination by type of ready-to-eat vegetables

\begin{tabular}{|c|c|c|}
\hline Ready-to-Eat vegetable & No. of samples & No. of unsatisfactory samples (\%) \\
\hline Arugula & 11 & $11(100 \%)$ \\
\hline Lettuce (sliced) & 47 & $40(85 \%)$ \\
\hline Cabbage (sliced/grated) & 24 & $16(67 \%)$ \\
\hline Salad mix & 34 & $22(65 \%)$ \\
\hline Cucumbers (sliced) & 22 & $12(55 \%)$ \\
\hline Other vegetables & 21 & $10(48 \%)$ \\
\hline Tomatoes + cucumbers (sliced) & 22 & $7(32 \%)$ \\
\hline Tomatoes (sliced) & 62 & $18(29 \%)$ \\
\hline Total number of samples & 243 & $136(56 \%)$ \\
\hline
\end{tabular}


Table 3. Prevalence of microorganisms in RTE vegetables

\begin{tabular}{|c|c|c|c|}
\hline Microorganism & No. of samples & $\begin{array}{c}\text { No. of unsatisfactory samples } \\
(\%)\end{array}$ & $\begin{array}{c}\text { Concentration range } \\
\log (\text { CFU/g) }\end{array}$ \\
\hline Yeasts/molds & 243 & $105(43.2 \%)$ & $3.60-5.81$ \\
\hline Enterobacteriaceae & 243 & $96(39.5 \%)$ & $3.20-6.48$ \\
\hline Coagulase-positive staphylococci & 243 & $12(4.9 \%)$ & $3.26-4.65$ \\
\hline E. coli & 243 & $4(1.6 \%)$ & $3.08-5.48$ \\
\hline Salmonella spp. & 243 & 0 & - \\
\hline Listeria monocytogenes & 243 & 0 & - \\
\hline Sulphite-reducing Clostridia & 243 & 0 & - \\
\hline
\end{tabular}

Our findings are consistent with the findings of a UK study by Sagoo et al., which reported that Enterobacteriaceae were present in levels above $10^{4}$ $\mathrm{CFU} / \mathrm{g}$ in $37 \%$ of the analyzed samples [22]. High levels of yeast/molds and Enterobacteriaceae are expected in raw vegetables as they are part of their natural microflora. Epiphytic microflora of raw vegetables predominantly consists of Enterobacteriaceae, such as Enterobacter and Erwinia spp. and also Pseudomonas spp. High levels of Enterobacteriaceae may also be derived from contamination with soil or as a result of poor handling during harvesting and packaging [23].

Study in Portugal also reported a poor quality of ready-to-eat vegetables. A high number of RTE vegetables presented poor microbiological quality (86\% aerobic mesophilic counts, $74 \%$ coliforms, $4 \%$ E. coli), despite the absence of pathogens [24].

In 2017, we have also examined 11 samples of packaged RTE leafy green vegetables. Seven out of 11 samples $(63.6 \%)$ were unsatisfactory due to contamination with Enterobacteriaceae (4-5 log CFU/g), although the products were labelled as ready-to-eat with no need for washing. This was a small number of analyzed samples but it showed that more studies are needed on microbiological quality of packaged RTE vegetables. The study by Nousiainen et al. showed that packaged RTE vegetables in Finland had very high counts of aerobic and coliform bacteria [25]. Study in Italy also reported that all analyzed samples of packaged RTE salads had aerobic mesophilic count above $10^{6}$ CFU/g [26]. Such microbial contamination fastens spoilage, thus decreasing product shelf-life.

The absence of Salmonella and Listeria monocytogenes in RTE vegetables from our study is consistent with other published studies [24-32].

A study conducted in Canada inspected 31,329 samples of fresh fruits and vegetables sold at retail (12,073 samples of leafy vegetables, 4,837 samples of tomatoes). Samples were analyzed for the presence of pathogens (Salmonella, Escherichia coli O157:H7, Shigella, Campylobacter and Listeria monocytogenes), as well as for E. coli, an indicator of fecal contamination. Results showed low prevalence of bacterial contamination. Salmonella and Listeria monocytogenes were detected in very low number of samples (only 2 and 14 samples out of all samples of leafy greens, respectively), while Escherichia coli O157:H7, Shigella and Campylobacter were not detected in any of the samples. E. coli was found at levels below $1 \%$ of all samples [27].

$E$. coli is considered the most suitable indicator of fecal contamination since it is found in warm-blooded animals as well as humans, and because it correlates best with potential contamination by enteric pathogens [28]. In our study, E. coli was detected in only four $(1.6 \%)$ samples. Similar results were reported by Johnston et al., who found extremely low levels of E. coli [29, 30]. Other studies reported much higher levels of E. coli. Olivera et al. reported that $E$. coli was detected in $53.1 \%$ of the RTE minimally processed vegetables in Brazil [31]. Study in Finland reported that $E$. coli was found in $15 \%$ of the leafy green vegetable samples [25], while study conducted in Maryland, USA reported that $10.65 \%$ of fresh produce were positive for E. coli [32]. E. coli O157:H7 was not included in the study since our epidemiological data does not support its presence in this food category.

Since RTE vegetables are raw minimally processed food, washing is the most critical step in assuring its safety. However, there is no completely effective washing strategy without affecting the quality of RTE vegetables. Food handlers usually use chlorine products for washing of fruit and vegetables. Although chlorine significantly reduces the microbial load on vegetables, its disadvantage is that it may produce unacceptably high levels of trihalomethans on heavily organically contaminated material (THMs) [33]. Taking this fact into consideration, the best disinfection is achieved when organic matter is eliminated beforehand [33].

\section{Conclusions}

Our study showed poor microbiological quality of RTE vegetables which brings certain concerns regarding the safety of these products. Although pathogens like $L$. monocytogenes and Salmonella spp. were not isolated, there is still very high and very frequent contamination with indicator microorganisms. This contamination implies that RTE vegetables aren't properly washed and prepared 
for consumption. Besides of safety concerns, such microbial contamination fastens spoilage, thus decreasing product shelf-life.

Proper washing and adequate hygiene during preparation are a must have prerequisite for food safety. Both, food handlers and consumers must adopt good hygiene practice to minimize the risks of foodborne diseases through RTE vegetables as vehicles.

More studies are needed regarding microbiological quality of packaged ready-to-eat vegetables, since their mislabeling is giving a customer an incorrect information.

\section{REFERENCES}

[1] EU (European Union). Regulation (EC) No 2073/2005 on microbiological criteria for foodstuffs. Off. J. Eur. Union 338 (1), 1-26, 2005.

[2] Republic of Croatia. Croatian National Guidelines on microbiological criteria for foodstuffs, Ministry of Agriculture, $3^{\text {rd }}$ edition, 2011.

[3] FAOSTAT 2008.http://www.fao.org/es/ess/yearbook/vol_1 _1/pdf/b03.pdf (accessed 10th August, 2017).

[4] FAO/WHO. Microbiological hazards in fresh leafy vegetables and herbs: Meeting Report. Microbiological Risk Assessment Series No. 14, Rome, 2008.

[5] EFSA (European Food Safety Authority). Scientific opinion on the risk posed by pathogens in food of non-animal origin. Part 1 (outbreak data analysis and risk ranking of food/pathogen combinations). EFSA J. 11, 3025, 2013.

[6] EFSA (European Food Safety Authority). Scientific opinion on the risk posed by pathogens in food of non-animal origin. Part 2 (Salmonella and norovirus in leafy greens eaten raw as salads). EFSA J. 12, 3600, 2014.

[7] Y. Pachepsky, D. R. Shelton, M. Jet, et al. Irrigation Water as a Source of Pathogenic Microorganisms in Produce: a review. Adv Agron 113, 73-138, 2011.

[8] Centers for Disease Control and Prevention. https://www. cdc. gov/ecoli/2018/o157h7-04-18/index. html (accessed 21st September 2018).

[9] L. Verhoeff-Bakkenes, H. A. P. M. Jansen, P. H. in't Veld, R. R. Beumer, M. H. Zwietering, F. M. van Leusden. Consumption of raw vegetables and fruits: a risk factor for Campylobacter infections. Int J Food Microbiol 144(3), 406-412, 2011.

[10] K. Holvoet, I. Sampers, M. Seynnaeve, L. Jacxsens, M. Uyttendaele. Agricultural and management practices and bacterial contamination in greenhouse versus open field lettuce production. Int J Environ Res Public Health 12(1), 32-63, 2014

[11] M. I. Khalid, J. Y. Tang, N. H. Baharuddin, N. S. Rahman, N. F. Rahimi, S. Radu. Prevalence, antibiogram, and cdt genes of toxigenic Campylobacter jejuni in salad style vegetables (ulam) at farms and retail outlets in Terengganu. J Food Prot 78(1), 65-71, 2015.
[12] H. Mohammadpour, E. Berizi, S. Hosseinzadeh, M. Majlesi, M. Zare. The prevalence of Campylobacter spp. in vegetables, fruits, and fresh produce: a systemic review and meta-analysis. Gut Pathog 10:41, 2018. https://doi.org/10.1186/s13099-018-0269-2.

[13] Centers for Disease Control and Prevention. https://www.cdc.gov/parasites/cyclosporiasis/outbreaks/201 8/b-071318/ (accessed 21st September 2018).

[14] ISO (International Organization for Standardization). ISO 6579-1:2017 - Microbiology of the food chain - Horizontal method for the detection, enumeration and serotyping of Salmonella - Part 1: Detection of Salmonella spp.

[15] ISO (International Organization for Standardization). ISO 11290-1:2017 - Microbiology of the food chain - Horizontal method for the detection and enumeration of Listeria monocytogenes and other Listeria spp. - Part 1: Detection method

[16] ISO (International Organization for Standardization). ISO 11290-2:2017 - Microbiology of the food chain - Horizontal method for the detection and enumeration of Listeria monocytogenes and other Listeria spp. - Part 1: Enumeration method

[17] ISO (International Organization for Standardization). ISO 21528-2: 2017 - Microbiology of the food chain - Horizontal method for the detection and enumeration of Enterobacteriaceae - Part 2: Colony-count technique

[18] ISO (International Organization for Standardization). ISO 16649-2:2001 - Microbiology of food and animal feeding stuffs - Horizontal method for the enumeration of beta-glucuronidase positive $E$. coli - Part 2: Colony-count technique at 44 degrees $\mathrm{C}$ using 5-bromo-4-chloro-3-indolyl beta-D-glucuronide

[19] ISO (International Organization for Standardization). ISO 6888-1:2004 - Microbiology of food and animal feeding stuffs - Horizontal method for the enumeration of coagulase-positive staphylococci (including Staphylococcus aureus and other species) - Part 1: Technique using Baird-Parker agar medium

[20] ISO (International Organization for Standardization). ISO 15213:2004 - Microbiology of food and animal feeding stuffs - Horizontal method for the enumeration of sulphite-reducing bacteria under anaerobic conditions

[21] ISO (International Organization for Standardization). ISO 21527-1:2012 - Microbiology of food and animal feeding stuffs - Horizontal method for the enumeration of yeasts and moulds - Part 1: Colony count technique in products with water activity greater than 0,95

[22] S. K. Sagoo, C. L. Little, L. Ward, I. A. Gillespie, R. T. Mitchell. Microbiological study of ready-to-eat salad vegetables from retail establishments uncovers a national outbreak of salmonellosis. J Food Prot 66, 403-409, 2003.

[23] C. L. Little, D. Roberts, E. Young, J. de Louvois. Microbiological quality of retail imported unprepared whole lettuces: A PHLS food working group study. J Food Prot 62: 325-328, 1999.

[24] J. Campos, J. Mourão, N. Pestana, L. Peixe, C. Novais, P. Antunes. Microbiological quality of ready-to-eat salads: An underestimated vehicle of bacteria and clinically relevant 
antibiotic resistance genes. Int J Food Microbiol 166, 464-470, 2013.

[25] L. L. Nousiainen, S. Joutsen, J. Lunden, M. L. Hänninen, M. Fredriksson-Ahomaa. Bacterial quality and safety of packaged fresh leafy vegetables at the retail level in Finland. Int J Food Microbiol; 232, 73-79, 2016.

[26] M. A. Coniglio, G. Faro, M. Marranzano. The importance of the Microbiological quality of Ready-to-Eat Salads from a Public Health Importance. J Food Process Technol 7:4, 2016. http://dx.doi.org/10.4172/2157-7110.1000577 (accessed 25 September 2018).

[27] N. Denis, H. Zhang, A. Leroux, R. Trudel, H. Bietlot. Prevalence and trends of bacterial contamination in fresh fruits and vegetables sold at retail in Canada. Food Control 67, 225-234, 2016.

[28] M. P. Doyle \& M. C. Erickson. Closing the door on the fecal coliform assay. Microbe 1(4), 162-163, 2006.

[29] L. M. Johnston, L. A. Jaykus, D. Moll, M. C. Martinez, J. Anciso, B. Mora, C. L. Moe. A field study of the microbiological quality of fresh produce. J Food Prot 68, 1840-1847, 2005.

[30] L. M. Johnston, L. A. Jaykus, D. Moll, J. Anciso, B. Mora, C. L. Moe. A field study of the microbiological quality of fresh produce of domestic and Mexican origin. Int $\mathrm{J}$ Food Microbiol 112, 83-95, 2006.

[31] M. A. de Oliveira, V. M. de Souza, A. M. M. Morato Bergamini, E. C. Pereira De Martinis. Microbiological quality of ready-to-eat minimally processed vegetables consumed in Brazil. Food Control 22, 1400-1403, 2011.

[32] R. C. Korir, S. Parveen, F. Hashem, J. Bowers. Microbiological quality of fresh produce obtained from retail stores on the Eastern Shore of Maryland, United States of America. J Food Microbiol 56, 29-34, 2016.

[33] M. I. Gil, M. V. Selma, F. López-Gálvez, A. Allende. Fresh-cut product sanitation and wash water disinfection: problems and solutions. Int J Food Microbiol 134, 37-45, 2009. 